UG-FT-60/96

hep-ph/9604287

March 1996

\title{
Scale dependence of quark mass matrices in models with flavor symmetries
}

\author{
J. A. Aguilar-Saavedra, M. Masip \\ Departamento de Física Teórica y del Cosmos \\ Universidad de Granada \\ 18071 Granada, Spain
}

\begin{abstract}
Numerical correlations between fermion masses and mixings could indicate the presence of a flavor symmetry at high energies. In general, the search for these correlations using low-energy data requires an estimate of leading-log radiative corrections. We present a complete analysis of the evolution between the electroweak and the grand unification scales of quark mass parameters in minimal supersymmetric models. We take $M_{t}=180 \mathrm{GeV}$ and consider all possible values of $\tan \beta$. We also analize the possibility that the top and/or the bottom Yukawa couplings result from an intermediate quasifixed point (QFP) of the equations. We show that the quark mixings of the third family do not have a QFP behaviour (in contrast to the masses, the renormalization of all the mixings is linear), and we evaluate the low-energy value of $V_{u b}$ which corresponds to $V_{u b}\left(M_{X}\right)=0$. Then we focus on the renormalization-group corrections to (i) typical relations obtained in models with flavor symmetries at the unification scale and (ii) a superstring-motivated pattern of quark mass matrices. We show that in most of the models the numerical prediction for $V_{u b}$ can be corrected in both directions (by varying $\tan \beta$ ) due to top or bottom radiative corrections.
\end{abstract}

\section{Introduction.}

The recent observation of the top quark, with a mass around $180 \mathrm{GeV}$ [1], allows a more complete analysis of the Yukawa sector of the standard model. In particular, it allows an evaluation of the running to higher energy scales of the parameters in that sector. The perturbative unification of the gauge couplings obtained in minimal supersymmetric (SUSY) scenarios suggests that the (nonsinglet) matter and gauge contents do not change up to energies around $10^{16} \mathrm{GeV}$. If that is the case, the first step to understand the flavor structure of the standard model is to evolve it up to those energies. We present in the first part of this article an updated and complete analysis of the evolution from low energies to the unification scale $M_{X}$ of the 10 physical parameters in the quark mass matrices (6 masses, 3 mixing angles, and the complex CKM phase) in the minimal SUSY extension of the standard model (MSSM) [2]. We will study in detail the behaviour of the mixing angles when the top and/or bottom Yukawa couplings $h_{t, b}$ are large at $M_{X}$. In this regime 
[3] the renormalization-group corrections focus any initial value of the coupling at $M_{X}$ to a narrow interval $\delta h_{t}$ (around $\left.h_{t}\left(M_{Z}\right) \approx 1.2\right)$ at low energy: $\left(\delta h_{t} / h_{t}\right)\left(M_{X}\right) \gg\left(\delta h_{t} / h_{t}\right)\left(M_{Z}\right)$. We will analyze how this nonlinear evolution of the couplings (quasifixed point (QFP) behaviour) affects the mixings with the third generation; in particular, we will find the low-energy value of the mixing $s_{13}$ (with $V_{u b}=s_{13} e^{-i \delta_{13}}$ ) that corresponds to $s_{13}=0$ at $M_{X}$.

On the other hand, the observed pattern of fermion masses and mixings does not look accidental, and suggests a symmetry in the Yukawa matrices as the origin of the hierarchies. Obviously, such a flavor symmetry should be formulated at the unification scale. Although the Yukawa matrices contain more free parameters than physical observables, it is not easy to find simple structures that are able to accommodate without need of fine tuning the measured values of masses and mixings. As a matter of fact, the authors in Ref. 淊 classify the symmetric quark mass matrices with a maximal number of texture zeroes, and find that only five textures are acceptable experimentally. These matrices predict correlations between mass parameters that can be expressed in a simple (approximate) form; we will analyze how the correlations run from $M_{X}$ to low energies. We will also analyze a particular scenario [5] derived from the heterotic string which has been recently proposed as the only realistic possibility among the models within its class. In this scenario (and also in the model proposed in [6], with one more texture zero than the cases in [四] the renormalization-group corrections could be essential to obtain a predicted mixing $V_{u b}$ within the experimental limits.

\section{Evolution of quark masses and mixings.}

The quark Yukawa sector of the MSSM can be expressed in terms of the superpotential

$$
P=h_{i j}^{u} H Q_{i} u_{j}^{c}+h_{i j}^{d} H^{\prime} Q_{i} d_{j}^{c}
$$

with $H \equiv\left(H^{+} H^{0}\right), H^{\prime} \equiv\left(\begin{array}{ll}H^{\prime 0} & H^{\prime-}\end{array}\right)$ and $Q_{i} \equiv\left(u_{i} d_{i}\right)$. In that sector there are 10 independent physical parameters: once the Yukawa matrices are diagonalized, only the eigenvalues ( 3 in the up and 3 in the down quark matrices) and the Cabibbo-KobayashiMaskawa (CKM) matrix (with 3 mixing angles and a complex phase) appear in the Lagrangian. The procedure to obtain these parameters at $M_{X}$ from the pole masses and the low-energy mixings is the following. For the heavier quarks, the perturbative pole mass $M_{i}$ is related to the running mass $m_{i}\left(M_{i}\right)$ in the $\overline{\mathrm{MS}}$ scheme by a simple expression [7] (this change of scheme is numerically important due to the large size of $\alpha_{s}$ ). Taking $M_{t}=180$ $\mathrm{GeV}$ and $M_{b}=4.7 \mathrm{GeV}$ we obtain

$$
m_{b}\left(M_{b}\right)=0.880 M_{b}, \quad m_{t}\left(M_{t}\right)=0.946 M_{t}
$$


The running masses of the lighter quarks are given at $1 \mathrm{GeV}$ 8]: $m_{u}=0.0056 \mathrm{GeV}$; $m_{d}=0.0099 \mathrm{GeV} ; m_{s}=0.199 \mathrm{GeV} ; m_{c}=1.35 \mathrm{GeV}$. The masses evolve up to the lightest Higgs scale $m_{h} \approx M_{Z}$ due to gauge interactions only; this is a factor 0.58 for the values at $1 \mathrm{GeV}$ and 0.75 for $m_{b}\left(M_{b}\right)$. It will be also convenient to run the top quark mass down to that scale; we obtain $m_{t}\left(M_{Z}\right)=1.05 m_{t}\left(M_{t}\right)$.

At $M_{Z}$ we find the Yukawa couplings to the lightest neutral Higgs $h$, which correspond to the Yukawas in the standard model (we assume $m_{h}=M_{Z} \ll m_{\text {susy }}=250$ $\mathrm{GeV})$

$$
\tilde{h}_{i j}^{u}=\frac{m_{u_{i}}}{174 \mathrm{GeV}} \delta_{i j}, \quad \tilde{h}_{i j}^{d}=\frac{m_{d_{i}}}{174 \mathrm{GeV}} V_{i j}^{*}
$$

being $V_{i j}$ the CKM matrix at $M_{Z}$. From $M_{Z}$ to $M_{t}$ these Yukawas evolve due to the gauge and the (much smaller) Yukawa interactions of the light fermions; between $M_{t}$ and $m_{\text {susy }}$ the top Yukawa corrections are also important. From $m_{\text {susy }}$ up to $M_{X} \approx 10^{16}$ $\mathrm{GeV}$ we include the interactions of the SUSY particles and the extra Higgs scalars (the standard model and MSSM renormalization group equations can be found in Refs. [9, 10] respectively). The Yukawa couplings to the two Higgs doublets are (at $m_{\text {susy }}$ )

$$
h_{i j}^{u}=\frac{\tilde{h}_{i j}^{u}}{\sin \beta}, \quad h_{i j}^{d}=\frac{\tilde{h}_{i j}^{d}}{\cos \beta}
$$

where $\tan \beta$ is the ratio of VEVs giving mass to down and up quarks. At $M_{X}$ we diagonalize the Yukawa matrices (we express the eigenvalues as $h_{i}$, with $i=u, d \ldots$ ) and find the CKM matrix. We will neglect corrections to mixing angles and light quark masses 11 proportional to the soft SUSY breaking parameters, although these corrections can be significant in the large $\tan \beta$ regime [12]. We present in Figs. 1, 2 the evolution of masses and mixings for values of $\tan \beta$ between 1.29 and 67.7 , which correspond respectively to $h_{t} / 4 \pi$ and $h_{b} / 4 \pi$ equal to 0.25 at $M_{X}$ (we analyze below in detail the quasifixed point regions). In Fig. 1 1 we plot $r_{i} \equiv m_{i}\left(M_{X}\right) / m_{i}\left(M_{Z}\right)$ for the six quarks (note that the ratios of masses and Yukawas coincide), whereas Fig. 2 expresses the ratios $r_{i j} \equiv V_{i j}\left(M_{X}\right) / V_{i j}\left(M_{Z}\right)$ $(i j=u s, c b, u b)$ and $r_{\delta} \equiv \arg \left[V_{u b}\left(M_{X}\right)\right] / \arg \left[V_{u b}\left(M_{Z}\right)\right]$ (in the Maiani parametrization). In order to compare the relative renormalization of the mixings and different ratios of masses, we also plot $R_{u}^{1 / 3}, R_{d}$ in Fig. 2, where $R_{u} \equiv \frac{m_{u, c}\left(M_{X}\right) / m_{t}\left(M_{X}\right)}{m_{u, c}\left(M_{Z}\right) / m_{t}\left(M_{Z}\right)}$ and $R_{d} \equiv \frac{m_{d, s}\left(M_{X}\right) / m_{b}\left(M_{X}\right)}{m_{d, s}\left(M_{Z}\right) / m_{b}\left(M_{Z}\right)}$. We take all the masses and mixings at $M_{Z}$ in their central value $\left(V_{u s}=0.221 \pm 0.003\right.$, $V_{c b}=0.040 \pm 0.008$ and $\left.\left|V_{u b}\right|=0.0035 \pm 0.0015\right)$, with $M_{t}=180 \mathrm{GeV}$ and $\arg \left(V_{u b}\right)=-\pi / 2$. From the analysis it follows that:

- The evolution of $m_{u}$ and $m_{c}$ coincide at the $0.04 \%$; the same happens for $m_{d}$ and $m_{s}(0.06 \%)$ and $\left|V_{u b}\right|$ and $V_{c b}(0.05 \%)$ 


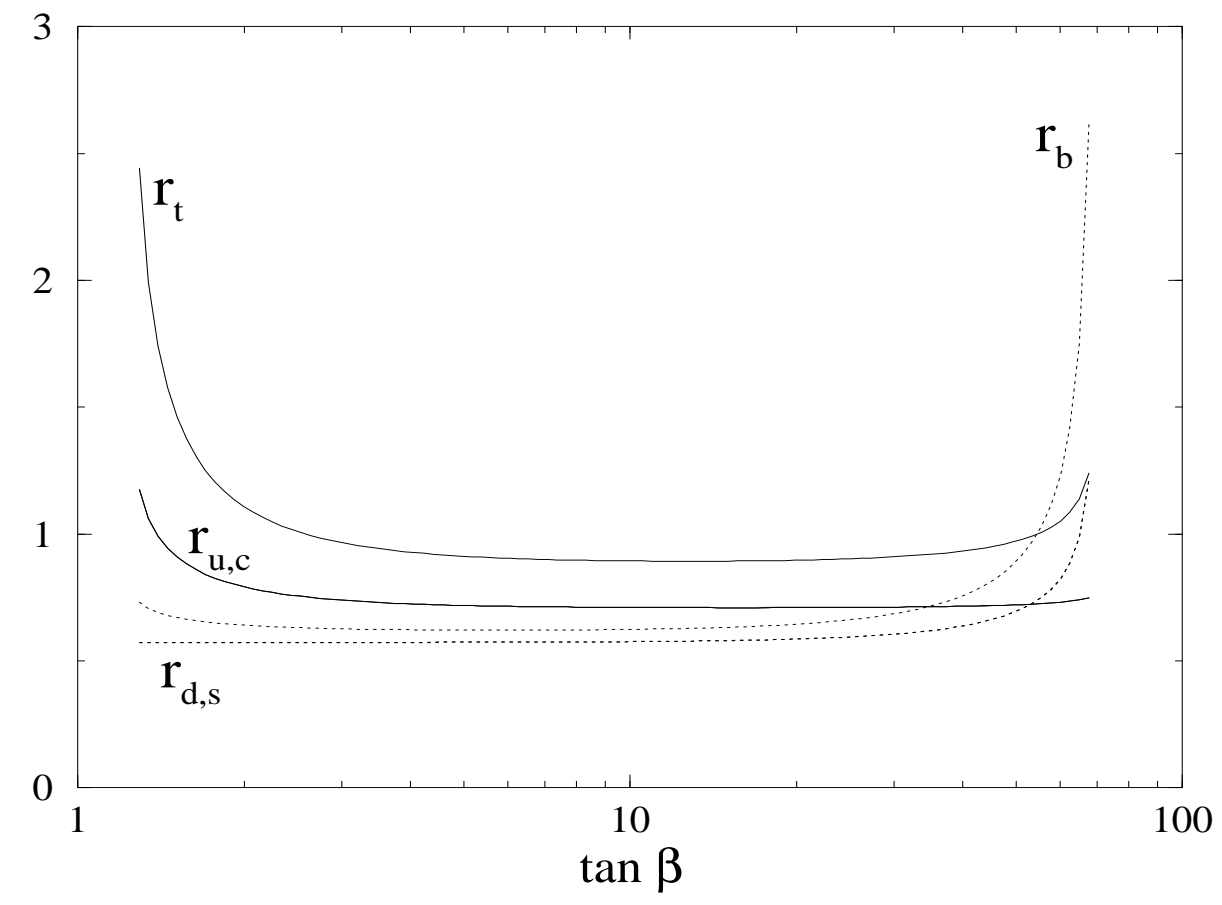

Figure 1: Ratios $r_{i}=m_{i}\left(M_{X}\right) / m_{i}\left(M_{Z}\right)(i=u, c, t, d, s, b)$ for different values of $\tan \beta$.

- The running of the Cabibbo mixing $V_{u s}$ and of the CKM phase (in $V_{u b}$ ) are smaller than the $0.03 \%$ and $0.07 \%$, respectively. The evolution of masses and mixings is insensitive to the value of the complex phase.

- The approximation [4]

$$
\begin{aligned}
\frac{V_{i j}\left(M_{X}\right)}{V_{i j}\left(M_{Z}\right)} & =\chi(i j=u s, c b, u b) \\
\frac{m_{u, c}\left(M_{X}\right) / m_{t}\left(M_{X}\right)}{m_{u, c}\left(M_{Z}\right) / m_{t}\left(M_{Z}\right)} & =\chi^{3} \\
\frac{m_{d, s}\left(M_{X}\right) / m_{b}\left(M_{X}\right)}{m_{d, s}\left(M_{Z}\right) / m_{b}\left(M_{Z}\right)} & =\chi
\end{aligned}
$$

is excellent (error smaller than 1\%) for $\tan \beta \leq 5$. These expressions, with $\chi=$ $\left(M_{X} / M_{Z}\right)^{\left(h_{t} / 4 \pi\right)^{2}}$, are obtained assuming that the top Yukawa coupling is dominant and constant between $M_{Z}$ and $M_{X}$. 


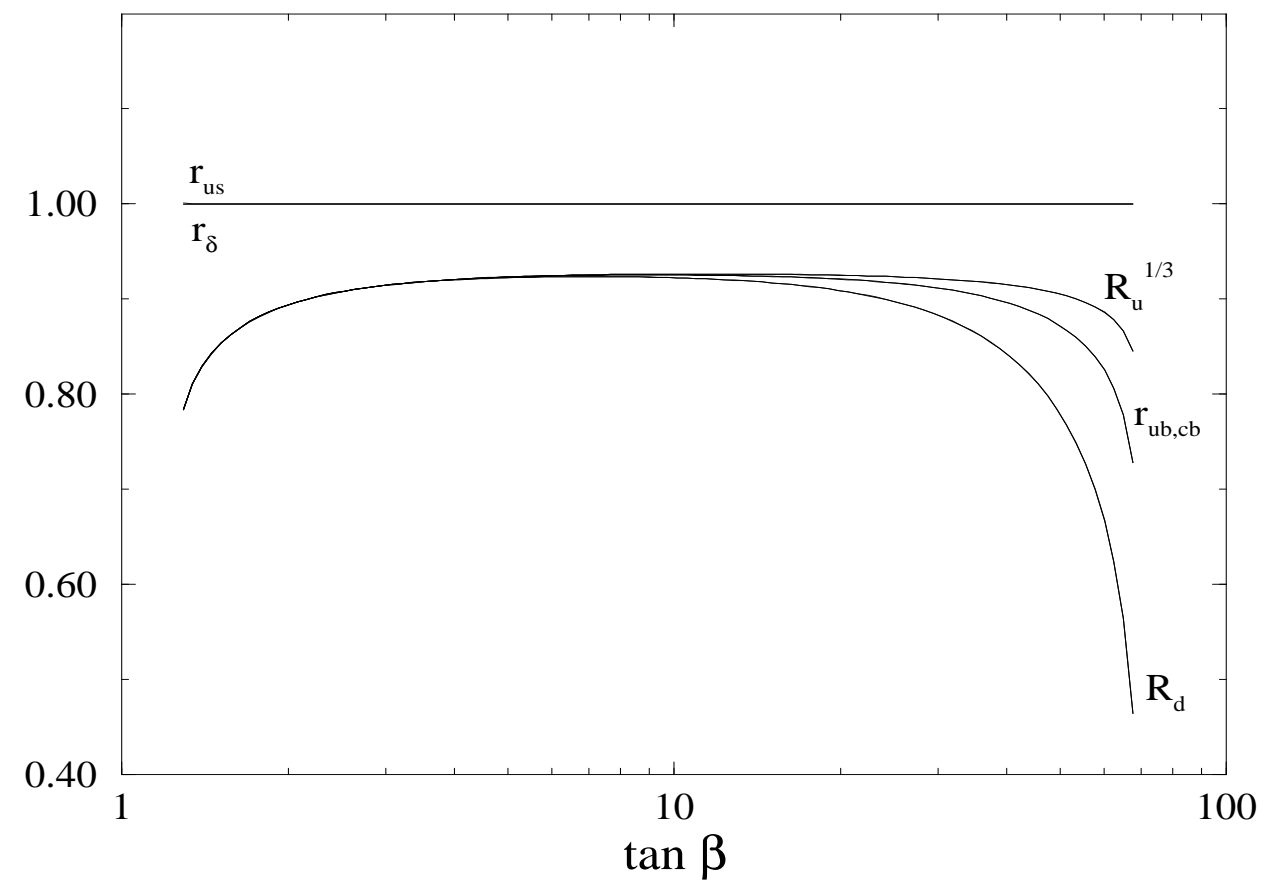

Figure 2: Ratios $r_{i j}=V_{i j}\left(M_{X}\right) / V_{i j}\left(M_{Z}\right)(i j=u s, c b, u b), r_{\delta}=\arg V_{u b}\left(M_{X}\right) / \arg V_{u b}\left(M_{Z}\right)$, $R_{u}=\frac{m_{u, c}\left(M_{X}\right) / m_{t}\left(M_{X}\right)}{m_{u, c}\left(M_{Z}\right) / m_{t}\left(M_{Z}\right)}$ and $R_{d} \equiv \frac{m_{d, s}\left(M_{X}\right) / m_{b}\left(M_{X}\right)}{m_{d, s}\left(M_{Z}\right) / m_{b}\left(M_{Z}\right)}$ for different values of $\tan \beta$.

As showed in [3], the low-energy value of a large Yukawa coupling could be related to an intermediate QFP (previous to the Pendleton-Ross infrared fixed point 13]) of the renormalization-group equations. The effect of the QFP would be to focus any large initial value (at $M_{X}$ ) of the coupling to a narrow region at $M_{Z}$. In the MSSM, the top coupling approaches a QFP value for $\tan \beta \approx 1$, and the bottom coupling may approach a QFP value for large $\tan \beta$. For a top mass on its upper experimental limit, both couplings could be related to QFPs [14]. In Tables 13 we show in some detail the behaviour of quark masses and mixings near the QFP. Table 11 expresses the initial values (at $M_{Z}$ ) of masses and mixings, whereas in Table 2 we write the values at $M_{X}$ for different values of $\tan \beta$ (which correspond to $h_{t}\left(M_{X}\right)=(2,8)\left[i . e ., h_{t}\left(M_{X}\right) / 4 \pi=(0.16,0.64)\right]$ and $\left.h_{b}\left(M_{X}\right)=(2,8)\right)$ for $M_{t}=180 \mathrm{GeV}$. In Table 3 both couplings are equal to 2 and/or 8 at $M_{X}$ (this implies $M_{t}=190-220 \mathrm{GeV}$ and $\left.\tan \beta=60-69\right)$. In all cases, the large Yukawa coupling at $M_{Z}$ varies very little around 1.2: $\left(\delta h_{t} / h_{t}\right)\left(M_{Z}\right) \approx 0.06$ for any value of $h_{t} / 4 \pi\left(M_{X}\right)$ larger than 0.32 . 


\begin{tabular}{cc}
\hline \hline$V_{u s}\left(M_{Z}\right)$ & $0.218-0.224$ \\
$\sqrt{m_{u} / m_{c}}\left(M_{Z}\right)$ & 0.0609 \\
$\sqrt{m_{d} / m_{s}}\left(M_{Z}\right)$ & 0.224 \\
\hline$V_{u b}\left(M_{Z}\right)$ & $0.002-0.005$ \\
$\sqrt{m_{u} / m_{t}}\left(M_{Z}\right)$ & 0.0040 \\
$\sqrt{m_{d} / m_{b}}\left(M_{Z}\right)$ & 0.043 \\
\hline$V_{c b}\left(M_{Z}\right)$ & $0.032-0.048$ \\
$\sqrt{m_{c} / m_{t}}\left(M_{Z}\right)$ & 0.066 \\
$\sqrt{m_{s} / m_{b}}\left(M_{Z}\right)$ & 0.193 \\
\hline \hline
\end{tabular}

Table 1: Experimental value at $M_{Z}$ of quark mass ratios (the central value) and CKM mixings (lower and upper limits).

The evolution of the mixing angles for Yukawa couplings near the QFP value presents the following features:

- The running of the mixings with the third family (and also the Cabibbo mixing) is highly linear, in the sense that $\left(\delta V_{i j} / V_{i j}\right)\left(M_{X}\right)=\left(\delta V_{i j} / V_{i j}\right)\left(M_{Z}\right)$, with $i j=$ $u s, u b, c b$. This fact means that the angles do not have a QFP behaviour. For example, for $h_{t}\left(M_{X}\right)=8$ the interval $V_{u b}\left(M_{Z}\right)=0.002-0.005$ evolves to $V_{u b}\left(M_{X}\right)=$ $0.00135-0.00337$.

- The evolution of $V_{u s}$ is still smaller than $0.04 \%$. The difference between the running of $V_{c b}$ and $V_{u b}$ and of the up and charm (down and strange) Yukawas, smaller than $0.02 \%$ and $0.04 \%(0.03 \%)$ respectively, do not grow when increasing the Yukawa couplings at $M_{X}$.

- The mixings with the third family $V_{i b}$ and the ratios $\sqrt{m_{u, c} / m_{t}}$ and $\sqrt{m_{d, s} / m_{b}}$ tend to zero at $M_{X}$ when $h_{t}\left(M_{X}\right)$ and/or $h_{b}\left(M_{X}\right)$ increase. However, when increasing for example $h_{t}$, the three quantities decrease at different rate. As a consequence, it will be always possible to correct a relation between masses and mixings by varying $\tan \beta$ and going to the adecuate (top or bottom) QFP region (see next section).

To illustrate the size of the nonlinear effects (first point above), we will consider the case when $V_{u b}$ vanishes at $M_{X}$. The running from $M_{X}$ to $M_{Z}$ generates then a nonzero 


\begin{tabular}{ccccc}
\hline \hline $\tan \beta$ & 1.22 & 1.44 & 65.0 & 69.5 \\
$h_{t}\left(M_{Z}\right)$ & 1.33 & 1.25 & 1.03 & 1.03 \\
$h_{b}\left(M_{Z}\right)$ & 0.028 & 0.031 & 1.16 & 1.24 \\
$h_{t}\left(M_{X}\right)$ & 8 & 2 & 1.17 & 1.51 \\
$h_{b}\left(M_{X}\right)$ & 0.024 & 0.021 & 2 & 8 \\
\hline$V_{u s}$ & $0.218-0.224$ & $0.218-0.224$ & $0.218-0.224$ & $0.218-0.224$ \\
$\sqrt{m_{u} / m_{c}}$ & 0.0609 & 0.0609 & 0.0609 & 0.0608 \\
$\sqrt{m_{d} / m_{s}}$ & 0.224 & 0.224 & 0.224 & 0.224 \\
\hline$V_{u b}$ & $0.00135-0.00337$ & $0.00178-0.0042$ & $0.00156-0.00390$ & $0.00125-0.00313$ \\
$\sqrt{m_{u} / m_{t}}$ & 0.00223 & 0.00310 & 0.00325 & 0.00289 \\
$\sqrt{m_{d} / m_{b}}$ & 0.0355 & 0.0396 & 0.0325 & 0.0236 \\
\hline$V_{c b}$ & $0.0216-0.0324$ & $0.0269-0.0403$ & $0.0249-0.0374$ & $0.0200-0.0301$ \\
$\sqrt{m_{c} / m_{t}}$ & 0.0366 & 0.0509 & 0.0533 & 0.0474 \\
$\sqrt{m_{s} / m_{b}}$ & 0.159 & 0.177 & 0.146 & 0.106 \\
\hline$V_{u b}\left(M_{Z}\right)$ & $6.5610^{-6}$ & $3.5410^{-6}$ & $1.3710^{-6}$ & $1.2810^{-6}$ \\
\hline \hline
\end{tabular}

Table 2: Value at $M_{X}$ of quark mass ratios and CKM mixings for values of $\tan \beta$ which correspond to large $h_{t}$ or $h_{b}$ at $M_{X}\left(M_{t}=180 \mathrm{GeV}\right)$. In the last line we write the value of $V_{u b}\left(M_{Z}\right)$ which would correspond to $V_{u b}\left(M_{X}\right)=0$.

mixing. In Fig. 3 we plot $V_{u b}\left(M_{Z}\right)$ for $\tan \beta$ between 1.29 and 67.7 , with the fermion masses and the rest of mixings in their central value. We find a value much smaller than the experimentally preferred, although it grows when $\tan \beta$ decreases (i.e., for a fixed $m_{t}$, $h_{t}\left(M_{X}\right)$ increases). We also show in Table 2 the low energy value of $V_{u b}$ which corresponds in each case to a vanishing mixing at $M_{X}$. Note that $V_{u b}$, the smallest mixing in the CKM matrix ( $s_{13}$ in the Maiani parametrization), is a physical parameter whose zero value at one loop is not protected by any symmetry. A flavor structure giving $V_{u b}=0$ at $M_{X}$ would be caracterized by $V_{u b}\left(M_{Z}\right) \approx 10^{-5}$. 


\begin{tabular}{ccccc}
\hline \hline$M_{t}$ & 203 & 220 & 193 & 214 \\
$\tan \beta$ & 63.5 & 59.8 & 68.8 & 66.8 \\
$h_{t}\left(M_{Z}\right)$ & 1.17 & 1.27 & 1.10 & 1.24 \\
$h_{b}\left(M_{Z}\right)$ & 1.13 & 1.07 & 1.23 & 1.19 \\
$h_{t}\left(M_{X}\right)$ & 2 & 8 & 2 & 8 \\
$h_{b}\left(M_{X}\right)$ & 2 & 2 & 8 & 8 \\
\hline$V_{u s}$ & $0.218-0.224$ & $0.218-0.224$ & $0.218-0.224$ & $0.218-0.224$ \\
$\sqrt{m_{u} / m_{c}}$ & 0.0609 & 0.0609 & 0.0609 & 0.0609 \\
$\sqrt{m_{d} / m_{s}}$ & 0.224 & 0.224 & 0.224 & 0.224 \\
\hline$V_{u b}$ & $0.00146-0.0036$ & $0.0012-0.00301$ & $0.00121-0.00303$ & $0.00101-0.00251$ \\
$\sqrt{m_{u} / m_{t}}$ & 0.00276 & 0.00191 & 0.00264 & 0.00183 \\
$\sqrt{m_{d} / m_{b}}$ & 0.0317 & 0.0295 & 0.0233 & 0.0218 \\
\hline$V_{c b}$ & $0.0234-0.0352$ & $0.0192-0.0289$ & $0.0194-0.0292$ & $0.0161-0.0242$ \\
$\sqrt{m_{c} / m_{t}}$ & 0.0453 & 0.0315 & 0.0435 & 0.0301 \\
$\sqrt{m_{s} / m_{b}}$ & 0.142 & 0.132 & 0.104 & 0.098 \\
\hline \hline
\end{tabular}

Table 3: Value at $M_{X}$ of quark mass ratios and CKM mixings for large $h_{t}$ and $h_{b}$ couplings at $M_{X}\left(M_{t}\right.$ not fixed).

\section{Evolution of flavor relations}

We proceed now to study how the evolution from $M_{X}$ to $M_{Z}$ affects the relations between quark mass parameters obtained in models with flavor symmetries at the unification scale. First we will consider the 5 symmetric patterns with a maximal number of texture zeroes found in Ref. [4]. These patterns depend on 7 complex parameters which, after phase redefinitions, are reduced to 7 moduli and 2 phases (3 phases in solution 2). The approximate analysis shows that it is possible to adjust the experimental masses and mixings without need of fine tuning. In particular, the absence of significant cancellations implies that the only role of the 2 complex phases in each pattern is to generate the CKM phase. As a consequence, the 7 moduli fit the 6 quark masses and 3 mixings giving two relations. 


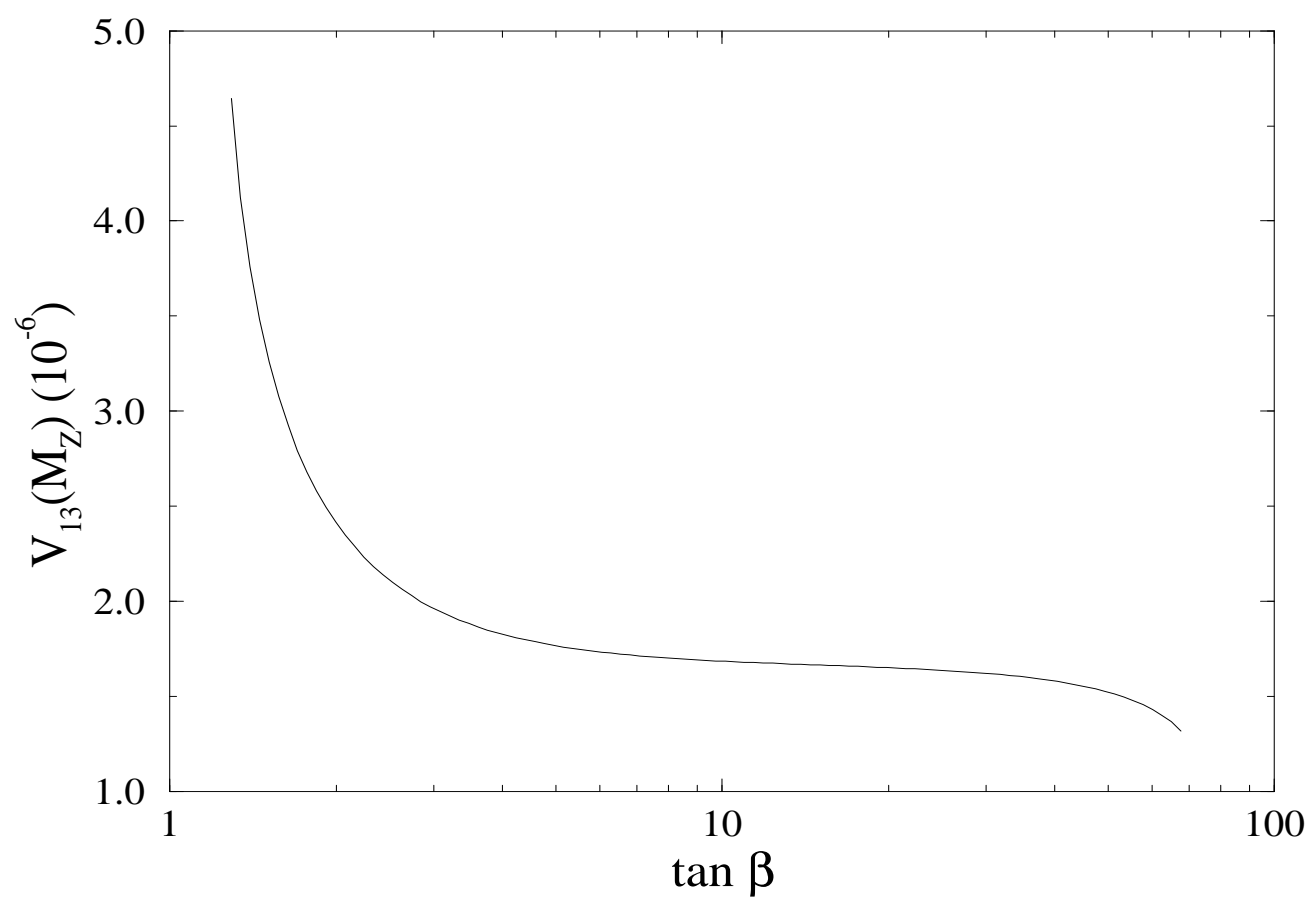

Figure 3: Value of $V_{u b}\left(M_{Z}\right)$ that would correspond to a zero value of $V_{u b}\left(M_{X}\right)$, plotted for different values of $\tan \beta$. The masses and the mixings $V_{u s}, V_{c b}$ are taken at their central value.

One relation [15] is shared by all the cases (solutions $1-5$ in 4 ):

$$
V_{u s}=\sqrt{\frac{m_{d}}{m_{s}}}
$$

(with complex corrections of modulus $\sqrt{m_{u} / m_{c}}$ in solutions $1,2,4,5$ ). The second relation is

$$
\frac{V_{u b}}{V_{c b}}=\sqrt{\frac{m_{u}}{m_{c}}}
$$

for solutions $1,2,4$ and

$$
V_{u b}=\sqrt{\frac{m_{u}}{m_{t}}}
$$

for solutions 3 and 5 (in the last case there are complex corrections of order $\frac{m_{t}}{2 m_{c}} V_{c b}^{2} \approx 20 \%$ ). The masses and the rest of the mixings can be adjusted to their central values, with an arbitrary CKM phase. 
Relations (8 10) are stablished at $M_{X}$, and one has to evolve the experimental quantities up to that scale in order to decide if they are acceptable. The running of the first two relations, however, is just a $0.2 \%$ (smaller than corrections to the approximate diagonalization of the matrices). Taking the masses in their central values we have $\sqrt{m_{d} / m_{s}}=0.223$ and $\sqrt{m_{u} / m_{c}}=0.064$, which compares well with the data $\left(V_{12}=0.221 \pm 0.003\right.$ and $V_{u b} / V_{c b}=0.08 \pm 0.02$ [B] $)$. The relation $V_{u b}=\sqrt{m_{u} / m_{t}}$ suffers sizeable renormalization-group corrections. At $M_{Z}$ we have $V_{u b}=0.0035 \pm 0.0015$ and $\sqrt{m_{u} / m_{t}} \approx 0.76 \sqrt{m_{u}(1 \mathrm{GeV}) / M_{t}}=0.0040$. The running from $M_{Z}$ to $M_{X}$ can be expressed in terms of the ratio

$$
r=\frac{\sqrt{m_{u} / m_{t}\left(M_{X}\right)}}{\sqrt{m_{u} / m_{t}\left(M_{Z}\right)}} / \frac{V_{u b}\left(M_{X}\right)}{V_{u b}\left(M_{Z}\right)}
$$

that we plot in Fig. $⿴$ for different values of $\tan \beta$. Note that for $\tan \beta<62, V_{13}$ diminishes less than $\sqrt{m_{u} / m_{t}}($ i.e., $r<1$ ), while for larger values of $\tan \beta$ we observe the opposite behaviour. If the masses and mixings were known with more accuracy, this fact could be used to correct the prediction

$$
V_{u b}=r 0.0040
$$

in the preferred direction.

The different running of the mixings and the ratios of quark masses involving the third family would also affect the symmetric texture with one more zero proposed in $[6]$. Those matrices predict $V_{c b}=\sqrt{m_{c} / m_{t}}$, a value that seems too large: the values of the masses at $M_{Z}$ suggest $V_{c b} \approx 0.76 \sqrt{m_{c}(1 \mathrm{GeV}) / M_{t}}=0.066$, while the experimental upper bound is 0.048 . Since the evolution of $V_{u b}\left(m_{u}\right)$ and $V_{c b}\left(m_{c}\right)$ coincide, the running from $M_{X}$ will be simply expressed by the same factor $r$ in Fig. 4 :

$$
V_{u b}=r 0.066
$$

and the relation would be experimentally acceptable for $\tan \beta \leq 1.4$ (with $M_{t}=180 \mathrm{GeV}$ ).

We will finally analyze a pattern of quark matrices derived from the heterotic string. The matrices have been proposed [5] as the only realistic possibility in a class of models compactified in the Tian-Yau manifold. Their structure is

$$
M_{u}=\left(\begin{array}{ccc}
0 & D_{0} & C_{0} \\
D_{0} & 0 & B_{0} \\
C_{0} & B_{0} & A_{0}
\end{array}\right), M_{d}=\left(\begin{array}{ccc}
D_{0}^{\prime} & 0 & 0 \\
0 & C_{0}^{\prime} & B_{0}^{\prime \prime} \\
0 & B_{0}^{\prime} & A_{0}^{\prime}
\end{array}\right)
$$

\footnotetext{
${ }^{1}$ We suppress an antisymmetric entry proportional to the VEVs of an extra Higgs doublet present in the model [f] since its presence would require a detailed analysis of flavor changing neutral currents.
} 


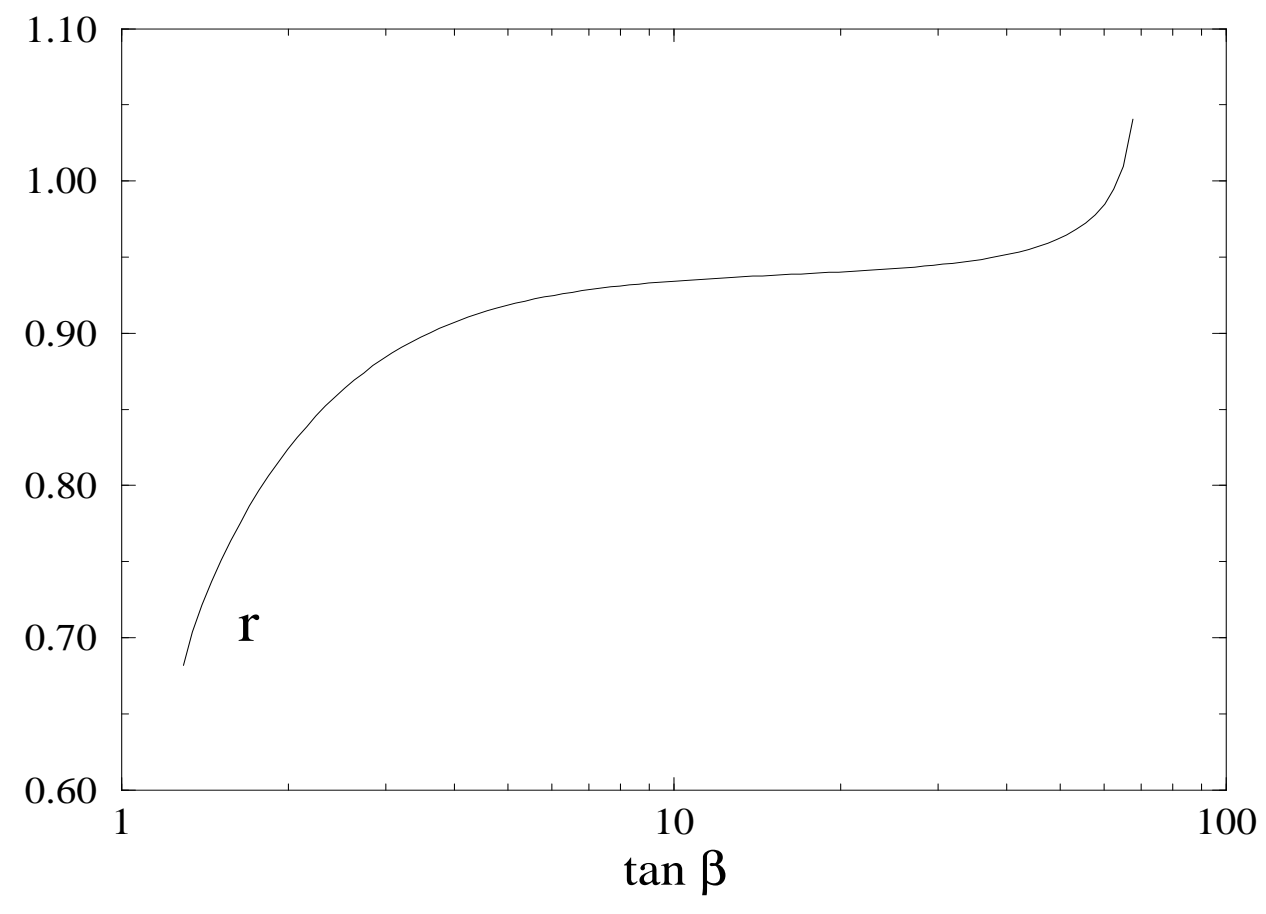

Figure 4: Ratio $r$ defined in (11) for different values of $\tan \beta$

By a redefinition of the quark fields we can put these matrices in a more convenient form:

$$
M_{u}=\left(\begin{array}{ccc}
0 & \tilde{D} & C \\
\tilde{D} & 0 & B \\
C & B & A
\end{array}\right), M_{d}=\left(\begin{array}{ccc}
D^{\prime} & 0 & 0 \\
0 & C^{\prime} & 0 \\
0 & \tilde{B}^{\prime} & A^{\prime}
\end{array}\right)
$$

where $\tilde{D}$ and $\tilde{B}^{\prime}$ are complex and the rest of the parameters are real and positive. As we will see, these 8 moduli and 2 phases can fit all the masses and the larger mixings to their central value and predict acceptable (but large) values for $V_{u b}$ and a nonzero (but small) complex CKM phase. The approximate diagonalization gives

$$
\begin{gathered}
m_{t}=A, \quad m_{c}=\frac{B^{2}}{A}, \quad m_{u}=\left|\frac{2 A B C \tilde{D}-A^{2} \tilde{D}^{2}}{A B^{2}}\right| \\
m_{b}=A^{\prime}, \quad m_{s}=C^{\prime}, \quad m_{d}=D^{\prime}
\end{gathered}
$$


and a CKM matrix (in the Maiani parametrization) with

$$
\begin{aligned}
V_{u s} & =\left|\frac{B C-A \tilde{D}}{B^{2}}\right| \\
V_{c b} & =\left|\frac{\tilde{B}^{\prime}}{A^{\prime}}-\frac{B}{A}\right| \\
\left|V_{u b}\right| & =\left|\frac{(B C-A \tilde{D}) \tilde{B}^{\prime}}{A B^{2}}-\frac{\tilde{D}}{B}\right|
\end{aligned}
$$

In terms of physical quantities we have

$$
V_{u b}=\left(V_{u s} V_{c b}+V_{12} \sqrt{\frac{m_{c}}{m_{t}}} e^{i \alpha}-\frac{m_{u} V_{c b}}{2 m_{c} V_{12}} e^{i \beta}\right)
$$

where $\alpha$ and $\beta$ are independent complex phases (the dominant phase $\alpha$ is related to the phase of $\left.\tilde{B}^{\prime}\right)$. At $M_{Z}$, for masses and mixings in their central values, the relation reads $V_{13}=0.0088+0.0146 e^{i \alpha}-0.0004 e^{i \beta}$. Then it seems that the small value of $V_{u b}$ requires a cancellation between the first two terms, with a best value $\left|V_{u b}\right|>0.0054$ (for $\alpha=\pi$ ). Renormalization-group corrections affect this relation due to the different running of $V_{c b}$ and $\sqrt{m_{c} / m_{t}}$, with the total effect captured again by the factor $r$ plotted in Fig. 目

$$
V_{u b}=0.0088+r 0.0146 e^{i \alpha}-0.00034 e^{i \beta}
$$

For low values of $r$, the lower bound for the predicted value of $\left|V_{u b}\right|$ decreases. For example, for $\tan \beta=1.5$ we have $\left|V_{u b}\right|>0.002$ and a CKM phase $\pi / 2 \leq \delta_{13} \leq 3 \pi / 2$, whereas $\left|V_{u b}\right|<0.005$ would imply $\tan \beta \leq 30$ (for all the quark masses and the rest of mixings in their central values). Note that for smaller values of $V_{c b}$, this bounds are relaxed.

\section{Conclusions}

The observed value of $M_{t}$ implies that $h_{t}$ is the dominant term in the renormalizationgroup equations at large scales. As a consequence, the corrections to the quark masses lose universality and there appear nontrivial corrections to the CKM mixings of the light quarks with the third family. In addition, the low-energy value of $h_{t}$ could be related to a QFP of the equations: any large value of $h_{t}\left(M_{X}\right)$ seems to converge to a narrow interval around 1.2 at $M_{Z}$. In the MSSM with $M_{t}=180 \mathrm{GeV}$ this forces a low value of $\tan \beta$, whereas an analogous situation occurs for $h_{b}$ in the large $\tan \beta$ regime. For $M_{t}$ around 200 $\mathrm{GeV}$ and large $\tan \beta$, both low-energy Yukawa couplings would result from any large value of the couplings at $M_{X}$ (a large value of $\tan \beta$ could be also motivated by the possibility to 
relax the $R_{b}$ anomaly [16]). In this framework, we perform an updated (with the new data for $M_{t}$ ) and complete (all values of $\tan \beta$ ) analysis of the evolution from $M_{Z}$ and $M_{X}$ of all the physical observables in the quark Yukawa sector of the MSSM. We study in detail the behaviour of the smallest CKM mixing $V_{u b}$ in the top and/or bottom QFP regions, and we show that the evolution is linear: $\delta V_{u b} / V_{u b}\left(M_{X}\right) \approx \delta V_{u b} / V_{u b}\left(M_{Z}\right)$. To illustrate the size the nonlinear corrections we analyze the value of $V_{u b}\left(M_{Z}\right)$ which corresponds to $V_{u b}\left(M_{X}\right)=0$; we obtain $V_{u b}\left(M_{Z}\right) \leq 10^{-5}$ (this value increases going to non-perturbative values of $h_{t}$ at $M_{X}$, i.e., lowering $\left.\tan \beta\right)$.

Then we analize the renormalization-group corrections to fermion mass relations which appear in models with flavor symmetries at $M_{X}$. In particular, we discuss the relations obtained for symmetric mass matrices with a maximal number of zeroes and in a superstring-motivated model. We show that in some relations the corrections can be numerically important (they are essential in some of the cases) and that they depend quite strongly on $\tan \beta$. In particular, for the relations analyzed the corrections can be expressed in terms of the ratio $r$ in (11). We find remarkable that, for a fixed $M_{t}, r$ goes to zero decreasing $\tan \beta$ and grows $(r>1)$ for $\tan \beta$ large $(\tan \beta \geq 62)$. If the masses and mixings were measured with more accuracy, this fact could be used to conveniently correct the relations by varying $\tan \beta$, whereas if the Higgs sector of the MSSM were observed and $\tan \beta$ fixed, it could be used to exclude some of the quark mass matrix models.

\section{Acknowledgements}

This work was partially supported by CICYT under contract AEN94-0936, by the Junta de Andalucía and by the European Union under contract CHRX-CT92-004. We thank F. del Aguila for useful comments.

\section{References}

[1] F. Abe et al., Phys. Rev. Lett. 74 (1995) 2626; S. Abachi et al., Phys. Rev. Lett. 74 (1995) 2632.

[2] For previous work, see for example M. Olechowski and S. Pokorski, Phys. Lett. B257 (1991) 388.

[3] C.T. Hill, Phys. Rev. D 24 (1981) 691.

[4] P. Ramond, R.G. Roberts and G.G. Ross, Nucl. Phys. B406 (1993) 19.

[5] F. del Aguila, M. Masip and L. da Mota, Nucl. Phys. B440 (1995) 3. 
[6] S. Dimopoulos, L.J. Hall and S. Raby, Phys. Rev. Lett. 68 (1992) 1984; Phys. Rev. D45 (1992) 4195.

[7] N. Gray, D.J. Broadhurst, W. Grafe and K. Schilcher, Z. Phys. C 48 (1990) 673.

[8] Review of Particle Properties, Phys. Rev. D50 (1994) 1173

[9] H. Arason et al., Phys. Rev. D 46 (1992) 3945.

[10] D.J. Castaño, E.J. Piard and P. Ramond, Phys. Rev. D49 (1994) 4882.

[11] N. Arkani-Hamed, H.C. Cheng and L.J. Hall, LBL preprint 37893, hep-ph/9512302, Dec. 1995.

[12] T. Blazek, S. Raby and S. Pokorski, Phys. Rev. D52 (1995) 4151.

[13] B. Pendleton and G.G. Ross, Phys. Lett. B98 (1981) 291.

[14] C.D. Froggatt, I.G. Knowles and R.G. Moorhouse, Phys. Lett. B298 (1993) 356.

[15] R. Gatto, G. Sartori and M. Tonin, Phys. Lett. B29 (1968) 128.

[16] See for example D. García, R.A. Jiménez and J. Solà, Phys. Lett. B347 (1995) 2737. 